\title{
Desarrollo de un modelo de motivación para explicar el flow disposicional y la ansiedad en bailarines profesionales
}

\author{
Development of a Motivation Model to Explain Dispositional \\ Flow and Anxiety in Professional Dancers
}

Recibido: febrero 10 de 2012 | Revisado: julio 1 de 2012 | Aceptado: agosto 2 de 2012

\author{
Diana Amado Alonso* \\ Pedro Antonio SÁnchez-Miguel \\ Francisco Miguel Leo Marcos \\ DAVID SÁNCHEZ-OLIVA \\ TOMÁs García-CALVO \\ Universidad de Extremadura, España
}

doi:10.11144/Javeriana.UPSY12-2.dmmf

Para citar este artículo: Amado, D., SánchezMiguel, P. A., Leo, F. M., Sánchez-Oliva, D. \& García-Calvo, T. (2013). Desarrollo de un modelo de motivación para explicar el flow disposicional y la ansiedad en bailarines profesionales. Universitas Psychologica, 12(2), 457-470.

\footnotetext{
Departamento de Didáctica de la Expresión Musical, Plástica y Corporal, Facultad de Ciencias del Deporte, Universidad de Extremadura. Avenida de la Universidad s/n, 10071, Cáceres. E-mails: diamal@unex.es, pesanchezm@unex.es,franmilema@unex.es, davidsanchez@unex.es, tgarciac@ unex.es
}

RESUMEN

El propósito del presente estudio fue aplicar un modelo de motivación en bailarines profesionales, para conocer los procesos motivacionales que pueden facilitar o dificultar la aparición de consecuencias emocionales como el flow disposicional o la ansiedad. Participaron en la investigación 332 bailarines de diferentes conservatorios españoles (293 mujeres y 39 hombres), con edades comprendidas entre los 12 y 45 años, que respondieron a diferentes cuestionarios. Los resultados del modelo revelaron que el clima tarea compañeros predecía la satisfacción de las tres necesidades psicológicas, mientras que el clima ego compañeros tan solo predijo la necesidad de competencia, siendo esta variable predictor positivo de la motivación intrínseca y negativo de la desmotivación. Por último, la motivación intrínseca se mostró como predictor positivo del flow disposicional y, en menor medida, de la ansiedad, mientras que la desmotivación predijo únicamente la ansiedad. Como conclusión se destaca que el modelo de motivación planteado se ajusta correctamente con una muestra de bailarines profesionales. Palabras clave autores

Ansiedad, bailarines profesionales, flow disposicional, modelo de ecuaciones, procesos motivacionales.

Palabras clave decriptores

Psicología del deporte, investigación cuantitativa, motivación.

\section{A B S T R A C T}

The aim of the current study was to apply a motivation model in professional dancers to know motivational processes that might help or make difficult the appearance of emotional consequences such as dispositional flow or anxiety. 332 dancers from different Spanish conservatories participated in the research ( 293 females and 39 males), ranging in age from 12 to 45 years old who filled several questionnaires. Model`s results showed that peer mastery climate predicted satisfaction of the three psychological needs and peer performance climate only predicted necessity of competence, being this variable a positive predictor of intrinsic motivation and a negative predictor of amotivation. Finally, intrinsic motivation was a dispositional flow predictor and, to a lesser extent, was an anxiety predictor, whereas amotivation only predicted anxiety. To conclude, we emphasize that motivation model was correctly adjusted for a professional dancers sample.

Key words authors

Anxiety, Professional Dancers, Dispositional Flow, Equations Model, Motivational Process. 
Key words plus

Sport Psychology, Quantitative Research, Motivation.

\section{Introducción}

La danza es una actividad que requiere afrontar un desafío, tener unas metas muy claras, proporcionar un feedback constante y conciso y sentir un elevado nivel de concentración, atención y control sobre la ejecución. Además, se caracteriza por trasladar a la persona hacia otra dimensión que va más allá de la realidad, de forma que sus sentimientos quedan al desnudo, produciéndose una pérdida de conciencia y una alteración en la percepción del tiempo, lo que hace que sea muy satisfactorio tanto para quien está bailando como para quien lo está observando (Hefferon \& Ollis, 2006), y esto es lo que hace que la danza se considere un arte, la capacidad de crear una experiencia estética. Esta experiencia consiste en entrar en juego con el mundo representado en la obra de arte y dejar entre paréntesis nuestra realidad para dejarnos arrastrar por la estética, realizando una lectura activa de dicha obra (Vergara, 2007).

Si analizamos con detenimiento cada uno de los factores anteriormente comentados, corresponden a las dimensiones del estado de flow o estado de rendimiento óptimo, concepto muy estudiado en el ámbito de la psicología del deporte que se asocia con actividades que tienen una naturaleza autotélica, agradable y divertida por sí misma y que hace que la persona quede abstraída en una actividad aislándose de cualquier otro pensamiento y emoción (Jackson \& Csikszentmihalyi, 1999). Por tanto, la danza por sí misma es un contexto idóneo para alcanzar este estado (Hefferon \& Ollis, 2006), caracterizado por actuaciones sobresalientes del individuo y por su relación con consecuencias positivas para el rendimiento, la satisfacción y el compromiso con una actividad (González-Cutre, Sicilia \& Moreno, 2009; Moreno, Cano, GonzálezCutre, Cervelló \& Ruiz, 2009). Cuando una persona tiende a experimentar con frecuencia el estado de flow, se denomina flow disposicional (Jackson, Thomas, Marsh \& Smethurst, 2001), lo cual se considera una capacidad que se puede adquirir de forma innata o mediante el aprendizaje y, por ello, se puede ver influenciada por factores internos de la propia persona o por factores externos que provienen del ambiente y del entorno que le rodea (Csikszentmihalyi, 1990).

Sin embargo, la práctica de la danza no solo puede conducir a consecuencias positivas como el estado de flow, sino que puede provocar una gran tensión, fruto de las exigencias físicas y psicológicas a las que se ven sometidos los bailarines a la hora de presentar una coreografía ante un público, pues además de estos factores técnicos y psicológicos, incide la estética del bailarín y de la pieza y el juicio de la crítica (Buckroyd, 2000). Esto puede causar culpabilidad, frustración y ansiedad (Taylor \& Taylor, 1995) entendiendo esta última como un constructo multidimensional en el que se deben distinguir tanto aspectos somáticos (manifestaciones fisiológicas) como cognitivos (pensamientos negativos) (Martens, Burton, Vealey, Bump \& Smith, 1990), de modo que ambos aspectos afectan de diferente forma al rendimiento (Krane, Joyce \& Rafeld, 1994).

Ambas consecuencias, pueden derivarse de una serie de antecedentes y procesos motivacionales que ya han sido estudiados en el ámbito de la psicología del deporte siendo el enfoque de la Teoría de la Autodeterminación (Deci \& Ryan, 2000; Ryan \& Deci, 2000) uno de los más empleados para esto. Partiendo de esta teoría, son numerosos los estudios que se han centrado en estudiar el Modelo Jerárquico de la Motivación de Vallerand $(2001,2007)$ en varios contextos. Este modelo plantea la existencia de una serie de factores sociales o antecedentes motivacionales que van a determinar la satisfacción de las necesidades psicológicas básicas de una persona y estas, a su vez, van a actuar como mediadores entre los antecedentes y el tipo de motivación mostrado hacia la práctica de una actividad, el cual puede oscilar desde el nivel más autodeterminado (motivación intrínseca) hasta el nivel menos autodeterminado (desmotivación), lo que va a derivar en una serie de consecuencias comportamentales, afectivas y cognitivas (Vallerand, 2001). 
Siguiendo este modelo, la presente investigación se centra en el contexto de la danza para estudiar los procesos motivacionales que conducen al flow disposicional y a la ansiedad, es decir, la incidencia de antecedentes como el clima motivacional sobre la percepción de autonomía, competencia y relaciones sociales y cómo estas necesidades actúan de mediadores entre estos antecedentes motivacionales y el tipo de motivación mostrado hacia la práctica de la danza, distinguiendo entre motivación intrínseca o desmotivación, lo que va a desembocar en consecuencias emocionales más adaptativas, como el flow disposicional o más desadaptativas, como la ansiedad.

En este sentido, refiriéndose a los antecedentes motivacionales, el clima motivacional es un conjunto de señales implícitas o explícitas (Ames, 1992) generadas en los entornos de intervención por los profesores, entrenadores, compañeros y padres (Maehr, 1984), que afectan a las metas de logro de una persona, las cuales pueden ser más orientadas a la tarea o más orientadas al ego. Así, se pueden distinguir dos tipos de climas motivacionales, el clima que implica a la tarea y el clima que implica al ego. En el clima tarea, el progreso relacionado con el rendimiento se rige por normas autorreferenciales y el aprendizaje cooperativo y el esfuerzo individual se ven acentuados, mientras que el clima ego se caracteriza por la comparación social y la rivalidad intraindividual (Ames, 1992; Newton, Duda \& Yin, 2000).

El clima motivacional se considera un factor social que incide sobre la motivación a través de la satisfacción de las necesidades de competencia, autonomía y relaciones sociales, de tal forma que a mayor satisfacción de estas necesidades, mayor motivación intrínseca o nivel de autodeterminación mostrará una persona y a menor satisfacción, mayor será la desmotivación (Deci \& Ryan, 2000; Ryan $\&$ Deci, 2000). La motivación intrínseca implica el compromiso en una actividad por el placer que se obtiene al realizarla, por ello suele aparecer relacionada con consecuencias positivas como la adherencia a la práctica (García-Calvo, Cervelló, Jiménez, Iglesias \& Moreno, 2010; Lim \& Wang, 2009; Ulrich-French \& Smith, 2009), el disfrute (Gar-
cía-Calvo, Sánchez-Oliva, Sánchez-Miguel, Leo \& Amado, 2012; Moreno, López de San Román, Martínez-Galindo, Alonso \& González-Cutre, 2008) o el estado de flow (Amado, Leo, SánchezMiguel, Sánchez-Oliva \& García-Calvo, 2011; Jackson, Kimiecik, Ford \& Marsh, 1998; Martin \& Cutler, 2002; Moreno, Cervelló \& González-Cutre, 2006; Russell, 2001; Sicilia, Águila, González-Cutre $\&$ Moreno-Murcia, 2011). Sin embargo, la desmotivación, se refiere a la ausencia de motivación intrínseca y extrínseca por una actividad y suele ir acompañada de consecuencias más negativas, de forma contraria a lo que ocurre con la motivación intrínseca (Tessier, Sarrazin \& Ntoumanis, 2010).

En esta línea, los estudios realizados sobre las relaciones que se establecen entre las variables que se contemplan en esta investigación, señalan una relación positiva entre el clima que implica a la tarea, la satisfacción de las necesidades psicológicas básicas y los niveles más elevados de autodeterminación, y una relación negativa entre este tipo de clima y los niveles más bajos de autodeterminación (Cox \& Williams, 2008; Ommundsen \& Kvalø, 2007; Quested \& Duda, 2009, 2010; Reinboth \& Duda, 2006; Weiss, Amorose \& Wilko, 2009). En relación al flow disposicional, las investigaciones reconocen que la percepción de un clima motivacional que implica a la tarea y al ego y la motivación autodeterminada, predicen el flow disposicional, siendo la predicción del clima tarea más elevada que la del clima ego (Moreno et al., 2009; Moreno, Cervelló \& González-Cutre, 2010). Por último, en lo que se refiere a la ansiedad estado previa a las actuaciones, algunos trabajos realizados en el ámbito deportivo han puesto de manifiesto que el clima motivacional que implica al ego se relaciona con la desmotivación y con la ansiedad estado (Cecchini, González, Carmona \& Contreras, 2004). De esta forma, en ciertos casos se ha identificado una relación positiva entre el clima ego y la ansiedad estado (NordinBates, Quested, Walker \& Redding, 2012), así como entre la desmotivación y dicha ansiedad (Brière, Vallerand, Blais \& Pelletier, 1995; Yli-Piipari, Watt, Jaakkola, Liukkonen \& Nurmi, 2009), mientras que en otros trabajos no han hallado ninguna relación entre estas variables (Montero, 2010). 
Así pues, el objetivo principal de este trabajo es aplicar un modelo de motivación en bailarines profesionales que permita conocer los procesos motivacionales que se relacionan con el flow disposicional y aquellos que están más vinculados con la ansiedad. De esta forma, la hipótesis que se plantea es que el clima tarea predecirá positivamente la satisfacción de las necesidades psicológicas básicas, mientras que el clima ego lo hará negativamente; asimismo la satisfacción de estas necesidades predecirá positivamente la motivación intrínseca y negativamente la desmotivación. Esta última, se asociará de forma positiva con la ansiedad y la motivación intrínseca con el flow disposicional.

\section{Método}

\section{Participantes}

La muestra de la investigación fue seleccionada mediante muestreo aleatorio simple y estuvo compuesta por 332 practicantes de danza clásica $(N=89)$, contemporánea $(N=159)$ y española $(\mathrm{N}=84)$ de diferentes escuelas y conservatorios profesionales en España. Los participantes eran de género femenino $(N=293)$ y masculino $(N=39)$, con edades comprendidas entre los 12 y los 45 años $(M=19.90 ; D E=6.90)$ y llevaban bailando entre 2 y 27 años $(M=7.33 ; D E=5.55)$.

\section{Instrumentos}

\section{Percepción del clima motivacional creado por el profesor}

Se utilizó una adaptación de la versión traducida al castellano por González-Cutre, Sicilia y Moreno (2008) del instrumento denominado Perceived Motivational Climate in Sport Questionnaire-2 ([PMCSQ-2]; Newton et al., 2000). Este cuestionario consta de 33 ítems encabezados por la frase "Durante las clases de danza..." y agrupados en dos factores principales: Clima Tarea (17 ítems, ej.: "El profesor cree que todos somos importantes para el éxito del grupo") y Clima Ego (16 ítems, ej.: "El profesor/a cree que solo los/as mejores contribuyen al éxito del grupo"). La consistencia interna se comprobó a través del coeficiente alfa de Cronbach, mostrando una puntuación de 0.88 para el Clima Tarea y de 0.93 para el Clima Ego.

\section{Percepción del clima motivacional creado por los compañeros}

Se empleó una adaptación de la versión traducida al castellano por Moreno, López, Martínez-Galindo, Alonso y González-Cutre (2007) del instrumento denominado Peer Motivational Climate in Youth Sport Questionnaire ([Peer MCYSQ]; Ntoumanis \& Vazou, 2005). Este cuestionario consta de 9 ítems encabezados por la frase "Mis compañeros de danza...", y agrupados en dos factores, el Clima Tarea (5 ítems, ej.: "Se divierten cuando practican con otros") y el Clima Ego (4 ítems, ej.: "Quieren ser los mejores del grupo"). El coeficiente alfa de Cronbach mostró valores de 0.82 para el Clima Tarea y 0.86 para el Clima Ego.

\section{Satisfacción de las necesidades psicológicas básicas}

Se utilizó una versión adaptada y resumida de la Escala de Mediadores Motivacionales en el Deporte ([EMMD]; González-Cutre et al., 2007). Tras realizar el análisis psicométrico consistente en un análisis factorial exploratorio y en un análisis de fiabilidad, 8 ítems iniciales no tuvieron una aceptada fiabilidad por lo que el cuestionario final está compuesto por 15 ítems que aparecen agrupados en tres factores. El primer factor hace referencia a la necesidad de Relaciones Sociales (5 ítems, ej.: "Realmente me gusta la gente con la que ensayo"), el segundo factor corresponde a la necesidad de Autonomía (5 ítems, ej.: "Se tiene en cuanta mi opinión a la hora de crear una coreografía") y el tercer factor se refiere a la Competencia Percibida (5 ítems, ej.: "Creo que estoy entre los más capaces cuando se trata de bailar"). En cuanto a la consistencia interna, se observaron valores de 0.83 para la necesidad de Relaciones Sociales, de 0.7 para la Competencia y de 0.63 para la Autonomía. 


\section{Tipo de motivación}

Se empleó la versión traducida al castellano por Moreno, Cervelló y Martínez (2007) del instrumento denominado Behavioral Regulation in Exercise Questionnaire-2 ([BREQ-2]; Markland \& Tobin, 2004), utilizando dos factores de los cinco que integran dicho cuestionario: la Motivación Intrínseca (4 ítems, ej.: "Porque creo que la danza es divertida") y la Desmotivación (4 ítems, ej.: "No tengo claro por qué debo seguir bailando"). El coeficiente alfa de Cronbach mostró valores de 0.7 para la Motivación Intrínseca y de 0.48 para la Desmotivación. En este estudio, la fiabilidad de la Desmotivación fue baja, a pesar de que en el instrumento original mostraba índices adecuados. En este sentido, dado el pequeño número de ítems que componen este último factor y las características de la muestra, tal como indican algunos autores, la validez interna podría ser aceptada (Hair, Anderson, Tatham \& Black, 1998; Lowenthal, 2001; Nunnally \& Bernstein, 1994).

\section{Flow disposicional}

Se utilizó una adaptación de la versión traducida al castellano por García-Calvo, Jiménez, Santos-Rosa, Reina y Cervelló (2008) del instrumento denominado Dispositional Flow Scale ([DFS]; Jackson et al., 1998). El cuestionario consta de un total de 36 ítems introducidos por el enunciado "Cuando bailas..." y agrupados en un factor principal, el Flow Disposicional, el cual mostró valores de 0.93 en el coeficiente alfa de Cronbach.

\section{Ansiedad}

Se empleó la versión revisada del instrumento denominado Competitive State Anxiety Inventory- 2 ([CSAI-2R];Cox, Martens \& Russell, 2003) validada al castellano por Andrade, Lois y Arce (2007). El cuestionario consta de 17 ítems encabezados por la frase "Antes de las actuaciones...", que se encuentran divididos en tres factores, la Ansiedad Estado Cognitiva (5 ítems, ej.: "Me preocupa no tener el mejor resultado"), la Ansiedad Estado Somática (7 ítems, ej.: "Mi cuerpo está tenso") y la Autoconfian- za (5 ítems, ej.: "Estoy seguro de poder superar este desafío"). La consistencia interna mostró valores de 0.73 para la Ansiedad Estado Cognitiva, de 0.81 para la Ansiedad Estado Somática y de 0.82 para la Autoconfianza.

Hay que señalar que se llevó a cabo una adaptación de todos los instrumentos que consistió en modificar la redacción de la frase introductoria y de ciertos términos en algunos de los ítems al ámbito de la danza, y las respuestas a estos cuestionarios fueron contestadas en una escala tipo Likert de 5 puntos.

\section{Procedimiento}

Para llevar a cabo la recogida de datos, se desarrolló un protocolo de actuación para que la obtención de datos fuera similar en todos los participantes implicados en la investigación. En primer lugar, se contactó con las diferentes escuelas y conservatorios de danza para solicitarles su participación, y se informó a los profesores y directores de los objetivos de la investigación. Posteriormente, una vez obtenidos los permisos pertinentes, se procedió a la toma de datos, informando a los alumnos que su participación era voluntaria y que las respuestas serían tratadas confidencialmente. Los participantes rellenaron los cuestionarios en el vestuario, sin la presencia del profesor, en un clima que les permitía concentrarse sin tener ningún tipo de distracción durante aproximadamente 30 minutos. Durante este tiempo, el investigador principal estuvo siempre presente para aclarar cualquier tipo de duda.

\section{Resultados}

\section{Análisis descriptivo y correlaciones bivariadas}

En la Tabla 1 se puede observar que las puntuaciones medias más elevadas corresponden a las variables vinculadas con consecuencias más positivas, de modo que los valores más altos pertenecen al clima que implica a la tarea, tanto el generado por el profesor como por los compañeros, los mediadores psicológicos, la motivación intrínseca y el flow 


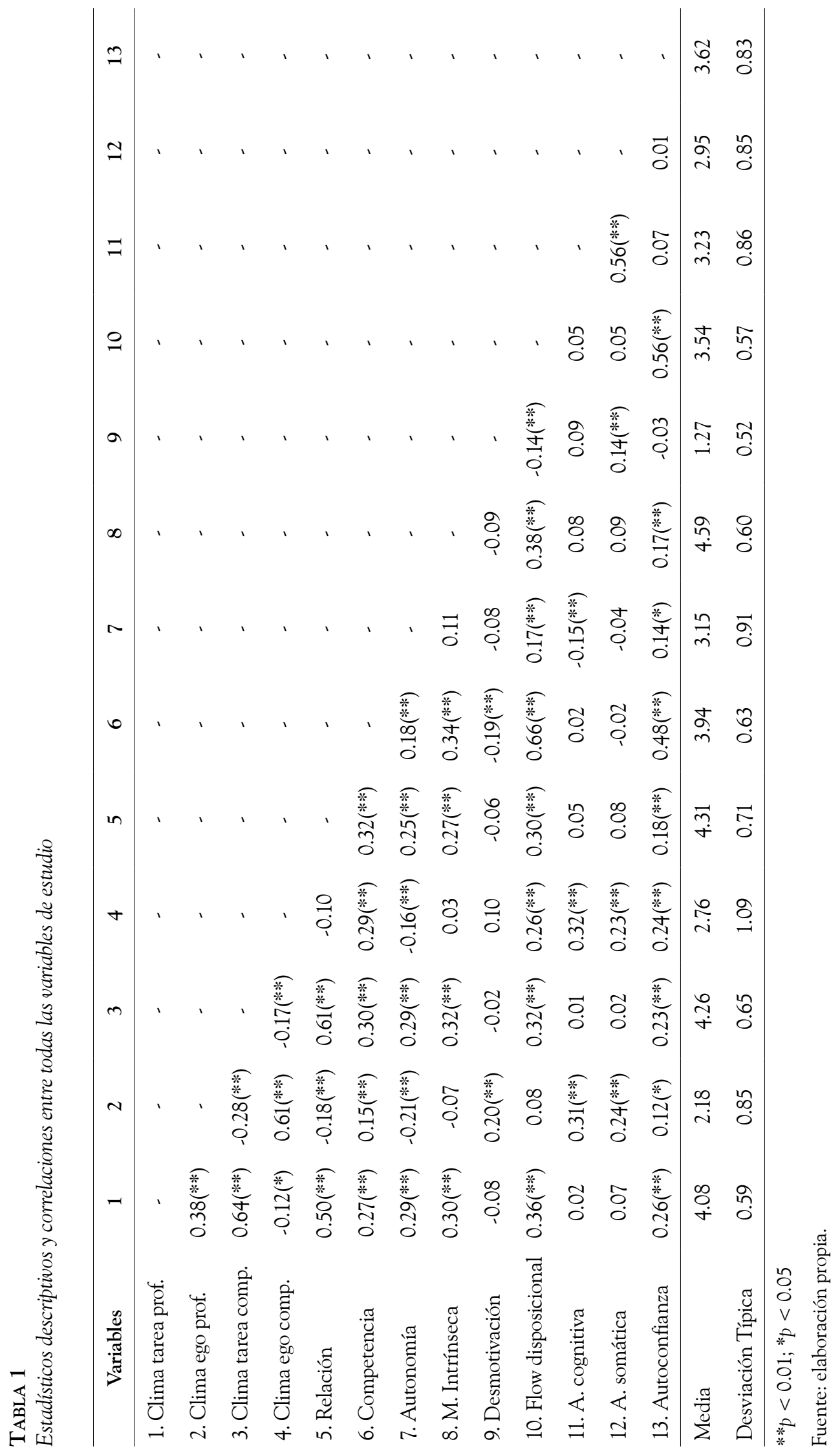


disposicional. Para concluir, en lo que respecta a la ansiedad previa a las actuaciones, se puede apreciar cómo el componente de autoconfianza es el que muestra mayor valor, seguido de la ansiedad cognitiva, cuya puntuación es más elevada que la ansiedad somática.

Además, en esta tabla aparecen reflejadas las relaciones existentes entre las diferentes variables a través del índice de correlación de Pearson. De esta forma, comenzando con los climas motivacionales, se observa que la percepción de un clima que implica a la tarea generado por el profesor y por los compañeros, se relaciona significativamente $(p<0.01)$ de forma positiva con los tres mediadores psicológicos, con la motivación intrínseca, con el flow disposicional y con la autoconfianza. En relación con el clima que implica al ego, cabe distinguir entre el creado por el profesor o el generado por los compañeros. El clima ego creado por el profesor se relaciona de forma negativa con las necesidades de relación y de autonomía y, de forma positiva, con la competencia, con la desmotivación, con la ansiedad cognitiva y somática y con la autoconfianza; pero estos resultados se deben interpretar con cautela porque únicamente la ansiedad cognitiva muestra un valor de correlación superior a 0.3. En cuanto al clima que implica al ego fomentado por los compañeros, se relaciona de forma positiva con la percepción de competencia, con el flow disposicional, con la ansiedad cognitiva y somática y con la autoconfianza, destacando las puntuaciones de la competencia y de la ansiedad cognitiva, con valores próximos o por encima del índice de correlación 0.3.

Respecto a los componentes de la teoría de la autodeterminación, se puede valorar cómo las necesidades de competencia y relaciones sociales así como la motivación intrínseca, se relacionan significativamente $(p<0.01)$ de forma positiva con el flow disposicional, destacando el valor de correlación de la competencia sobre todas las demás variables. Por último, en relación con el flow disposicional, se puede contemplar la existencia de una relación significativa $(p<0.01)$ y positiva con la autoconfianza, mientras que con la ansiedad cognitiva y somática no presenta relación.

\section{Modelo de ecuaciones estructurales}

Con el objetivo de comprobar los niveles de predicción mostrados por las diferentes variables incluidas en este trabajo se decidió realizar un modelo de ecuaciones estructurales, mediante el cual se tratará de testar un modelo teórico y establecer relaciones de direccionalidad con niveles de predicción en las distintas variables (Figura 1). Para realizar este análisis se utilizó el método de estimación de máxima verosimilitud y el procedimiento bootstrapping (Byrne, 2001). En dicho análisis, se realizó un modelo de medición con 11 constructos latentes (factores) que agrupaban 22 medidas observadas (ítems), de forma que los ítems de cada factor se agruparon en dos grupos (MacCallum \& Austin, 2000).

El modelo de ecuaciones estructurales fue elaborado con base en el Modelo Jerárquico de la Motivación de Vallerand (2001), incluyendo la percepción de los bailarines del clima motivacional generado por el profesor y por los compañeros como factor social, la satisfacción de las necesidades de competencia, autonomía y relaciones sociales como mediadores psicológicos entre los factores sociales y el tipo de motivación mostrado hacia la danza y por último, las consecuencias derivadas del tipo de motivación. Así pues, los resultados obtenidos tras desarrollar este modelo muestran que el clima tarea y el clima ego generado por el profesor no predicen la satisfacción de las necesidades psicológicas básicas. Sin embargo, el clima tarea creado por los compañeros predice positivamente la percepción de competencia, autonomía y relaciones sociales y el clima ego compañeros predice de esta misma forma la necesidad de competencia.

Siguiendo con el modelo, en la Figura 1 se puede observar cómo la percepción de competencia predice significativamente de forma positiva la motivación intrínseca y de forma negativa la desmotivación. Asimismo, se pone de manifiesto que la motivación intrínseca predice positivamente el flow disposicional y la ansiedad, aunque presenta mayor capacidad predictiva sobre el flow disposicional (0.87) que sobre la ansiedad (0.21), mientras que la desmotivación predice positiva y significativamente la ansiedad con una capacidad 
predictiva ligeramente superior a la de la motivación intrínseca (0.27).

Atendiendo a la validez del modelo, se consideraron diferentes índices de bondad: $\chi^{2}$ (Chi-Square and his significance), $\chi^{2} / g l$ (Chi-Square/degrees of freedom), CFI (Comparative Fit Index), TLI (TukerLewis Index), IFI (Incremental Fit Index), RMSEA (Root Mean Square Error of Approximation) y SR-
MR (Standardized Root Mean Residual). En relación con esto, los índices obtenidos fueron los siguientes: $\chi^{2}=457.43 ; g l=181 ; p<0.01 ; \chi^{2} / g l=2.53$; $\mathrm{CFI}=0.92 ; \mathrm{TLI}=0.9 ; \mathrm{IFI}=0.92 ; \mathrm{RMSEA}=0.07$; SRMR $=0.07$, lo que refleja que el modelo muestra unos valores de ajuste adecuados, pues el $\chi^{2} / g l$ presenta un valor por debajo de 4 (Hu \& Bentler, 1999), los índices incrementales CFI, TLI e IFI

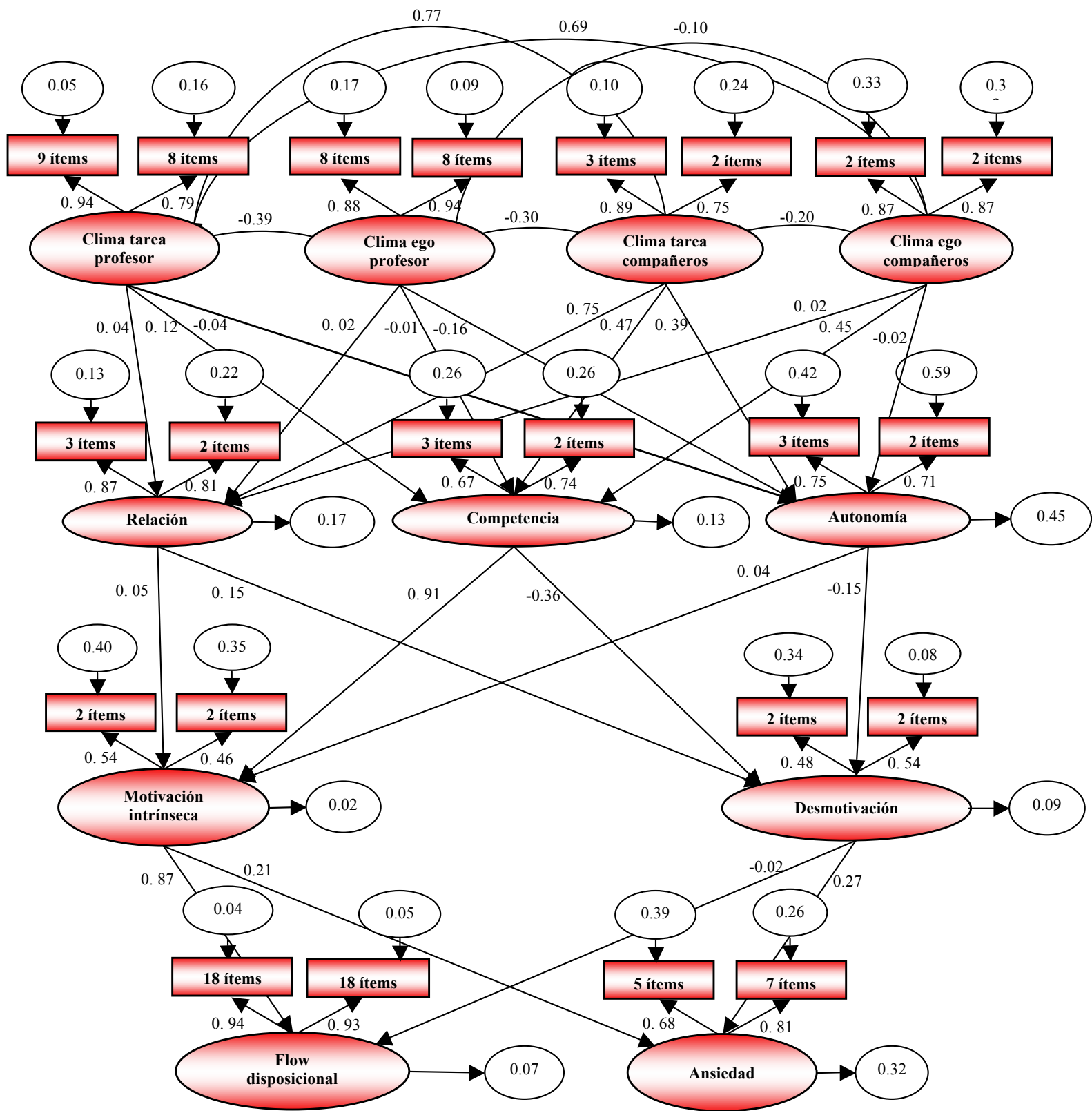

Figura 1. Modelo de ecuaciones estructurales que incluye el clima motivacional, los mediadores psicológicos, el tipo de motivación, el flow disposicional y la ansiedad.

Fuente: elaboración propia. 
muestran valores iguales o superiores a $0.9(\mathrm{Hu}$ $\&$ Bentler, 1995) y los índices de error RMSEA y SRMR presentan valores por debajo de $0.08(\mathrm{Hu}$ \& Bentler, 1999).

\section{Discusión}

El objetivo principal del presente trabajo fue aplicar un modelo de motivación en bailarines profesionales para conocer las variables que pueden facilitar o dificultar la aparición de ciertas consecuencias emocionales como el flow disposicional o la ansiedad, que suelen determinar la satisfacción y la adherencia con la práctica de una actividad. Para esto, se desarrolló un modelo de ecuaciones estructurales compuesto de cuatro niveles de predicción: el primer nivel es la percepción del clima motivacional generado por el profesor y por los compañeros, el segundo nivel es la satisfacción de las necesidades psicológicas básicas, el tercer nivel es el tipo de motivación mostrado hacia la danza y el cuarto y último nivel lo forman el flow disposicional y la ansiedad.

En este sentido, los resultados señalan que la percepción de un clima que implica a la tarea generado por los compañeros predice la satisfacción de las tres necesidades psicológicas básicas y que la percepción de un clima que implica al ego creado por estos, predice la satisfacción de la necesidad de competencia; en cambio, la percepción de un clima tarea o ego creado por el profesor no predice la satisfacción de estas necesidades. Esto sugiere el valor que conceden los bailarines a la figura que representan sus compañeros, pues incide directamente sobre la satisfacción de sus necesidades psicológicas básicas y, en definitiva, sobre su motivación hacia la danza.

Tras revisar los estudios realizados en psicología del deporte que utilizan el modelo de ecuaciones estructurales para conocer la capacidad de predicción de los climas motivacionales sobre las necesidades psicológicas básicas, cabe destacar que los trabajos realizados en el ámbito de la danza (Quested \& Duda, 2009, 2010) y en el terreno deportivo (Reinboth \& Duda, 2006) también han encontrado que el clima que implica a la tarea predice la satisfacción de las necesidades psicológicas básicas. En relación con el clima que implica al ego, autores como Almagro, Saénz-López, González-Cutre y Moreno-Murcia (2011) y Standage, Duda y Ntoumanis (2003) hallaron resultados similares a los encontrados en la presente investigación, señalando que el clima ego predecía la percepción de competencia. En la misma línea, algunos autores como González-Cutre et al. (2009) y Sproule, Wang, Morgan, McNeills y McMorris (2007) han encontrado que tanto el clima que implica a la tarea como el clima que implica al ego, predicen la percepción de competencia.

Siguiendo con el análisis de los resultados, se pone de manifiesto que la necesidad de competencia actúa como mediador entre el clima motivacional y el tipo de motivación, prediciendo de forma positiva la motivación intrínseca y de forma negativa la desmotivación. Este hecho puede verse influenciado porque en el ámbito de la danza profesional todos los trabajos siempre van destinados a conseguir mayor competencia motriz entendida como un "poder hacer eficaz" ante una determinada intención, lo que implica un entrenamiento concreto de la técnica como medio para dotar al bailarín de las herramientas necesarias que le permitan una suficiente autonomía para exteriorizar sus emociones (Fructuoso \& Gómez, 2001). Es decir, la técnica se utiliza como medio y no como fin, convirtiendo al cuerpo en un instrumento dotado de la mayor riqueza motriz, basada en la mejora de la coordinación neuromuscular, en el desarrollo del sentido espacial y rítmico y, en general, en la mejora de las capacidades físicas (Fructuoso \& Gómez, 2001). Estos resultados van en consonancia con los hallados en el ámbito deportivo por Montero (2010) y por Brunet y Sabiston (2009), en contraste con otros estudios que destacan la predicción positiva de las tres necesidades psicológicas básicas sobre la motivación intrínseca (Ntoumanis, 2005; Standage, Duda \& Pensgaard, 2005).

Por último, el modelo destaca la predicción positiva de la motivación intrínseca sobre el flow disposicional y la ansiedad, aunque el mayor peso predictivo recae sobre el flow disposicional. Esto implica que los bailarines que posean una motivación por la propia actividad y la satisfacción que 
les aporta su práctica, van a tener una elevada disposición a experimentar el estado de flow durante una situación de rendimiento, pero también van a tener probabilidad de percibir ansiedad ante esta situación. Estos hallazgos podrían deberse al nivel de autoexigencia que se imponen los bailarines, fruto de las peculiaridades del entorno en el que se desenvuelve la danza, lo que hace que aunque estén muy motivados por la propia actividad y su práctica les produzca mucha satisfacción, el hecho de representar sus trabajos ante un público siga siendo una fuente de ansiedad para ellos. Así pues, en el ámbito artístico a diferencia de lo que ocurre en el terreno deportivo, autores como Kirchner, Bloom y Skutnick-Henley (2008) analizaron las relaciones entre la ansiedad de rendimiento y el estado de flow en una muestra de 90 estudiantes de música, y hallaron que la propensión para experimentar el estado de flow así como la capacidad para tocar un instrumento o para cantar se relacionaba de forma significativa y positiva con la ansiedad de rendimiento. Estos resultados sugieren que tanto la ansiedad de rendimiento como el estado de flow, pueden existir simultáneamente, pues parece que la creación de las condiciones que llevan a alcanzar el estado de flow puede ser una estrategia muy útil para aliviar la intensidad de la ansiedad.

No obstante, es necesario destacar, cómo los resultados apuntan que la desmotivación predice en mayor medida la ansiedad que la motivación intrínseca, tal como indican algunos estudios realizados en el ámbito de la actividad física y el deporte, que relacionan la ansiedad con la desmotivación (Brière et al., 1995; Cecchini et al., 2004; Yli-Piipari et al., 2009) y consideran que es un estado negativo (Brunet \& Sabiston, 2009; Montero, 2010; ThogersenNtoumani \& Ntoumanis, 2006; Valle et al., 2009) contrario a la experimentación de consecuencias positivas como el estado de flow (Jackson et al., 2001; López-Torres, Torregrosa \& Roca, 2007).

Por tanto, con base en los resultados obtenidos, se puede confirmar la hipótesis del estudio que afirmaba que el clima tarea predeciría positivamente la satisfacción de las necesidades psicológicas básicas, mientras que el clima ego lo haría negativamente; asimismo la satisfacción de estas necesidades pre- deciría positivamente la motivación intrínseca y negativamente la desmotivación. Esta última, se asociaría de forma positiva con la ansiedad y la motivación intrínseca con el flow disposicional.

En este sentido, la principal conclusión que se puede extraer de esta investigación es que el modelo de motivación que se ha aplicado es válido y fiable para explicar los procesos motivacionales que se desarrollan en el ámbito de la danza profesional y que pueden desembocar en consecuencias emocionales como el flow disposicional o la ansiedad, que pueden ser muy determinantes para la satisfacción y el rendimiento en este ámbito, al igual que se ha demostrado en el contexto deportivo.

En relación con las limitaciones encontradas, en el presente estudio no se utilizan todos los constructos pertenecientes a un mismo marco teórico, sino que se combinan constructos de la Teoría de las Metas de Logro y de la Teoría de la Autodeterminación, de modo que los climas o antecedentes motivacionales pertenecen a la Teoría de las Metas de Logro, mientras que las necesidades psicológicas básicas y los tipos de motivación se abordan desde la Teoría de la Autodeterminación, lo que podría haber causado que los índices de ajuste del modelo no resultasen adecuados. Asimismo, desde el punto de vista metodológico, cabe destacar que el BREQ-2, a pesar de ser un instrumento validado para medir el tipo de motivación en contextos de ejercicio físico, el índice de fiabilidad encontrado para el factor desmotivación con esta muestra fue bajo.

Por esto, en un futuro se estima oportuno diseñar instrumentos específicos para el ámbito de la danza, que atiendan a diferentes dimensiones de la misma: profesional, recreativa, educativa, etc., con el fin de que las investigaciones que se realicen en torno a esta actividad puedan utilizar instrumentos propios sin tener que recurrir a los existentes en otros contextos de actividad física para adaptarlos a las peculiaridades de la danza. Del mismo modo, sería necesario valorar en posteriores trabajos los antecedentes motivacionales desde la óptica de la Teoría de la Autodeterminación, midiendo el apoyo del profesor o de los compañeros a las necesidades psicológicas básicas como factores sociales, en lugar 
de emplear los climas motivacionales que se encuadran en la Teoría de las Metas de Logro.

\section{Referencias}

Almagro, B. J., Saénz-López, P., González-Cutre., D. \& Moreno-Murcia, J. A. (2011). Clima motivacional percibido, necesidades psicológicas y motivación intrínseca como predictores del compromiso deportivo en adolescentes. Revista Internacional de Ciencias del Deporte, 25(7), 250-265.

Amado, D., Leo, F. M., Sánchez-Miguel, P. A., SánchezOliva, D. \& García-Calvo, T. (2011). Interacción de la teoría de la autodeterminación en la fluidez disposicional en practicantes de danza. Cuadernos de Psicología del Deporte, 11(1), 7-17.

Ames, C. (1992). Achievement goals, motivational climate, and motivational processes. En G. Roberts (Ed.), Motivation in sport and exercise (pp. 161-176). Champaign, IL: Human Kinetics.

Andrade, E., Lois, G. \& Arce, C. (2007). Propiedades psicométricas de la versión española del Inventario de Ansiedad Competitiva CSAI-2R en deportistas. Psicothema, 19(1), 150-155.

Buckroyd, P. (2000). The student dancer: Emotional aspects of the teaching and learning of dance. London: Dance Books.

Brière, N. M., Vallerand, R. J., Blais, M. R. \& Pelletier, L. G. (1995). Développement et validation d'une mesure de motivation intrinsèque, extrinsèque et d'amotivation en contexte sportif: L'Échelle de Motivation dans les Sports (EMS). International Journal of Sport Psychology, 26(4), 465-489.

Brunet, J. \& Sabiston, C. M. (2009). Social physique anxiety and physical activity: A self-determination theory perspective. Psychology of Sport and Exercise, 10(3), 329-335.

Byrne, B. M. (2001). Structural equation modeling with Amos: Basic concepts, applications, and programming. Mahwah, NJ: Erlbaum.

Cecchini, J. A., González, C., Carmona, M. \& Contreras, O. (2004). Relaciones entre el clima motivacional, la orientación de meta, la motivación intrínseca, la autoconfianza, la ansiedad y el estado de ánimo en jóvenes deportistas. Psicothema, 16(1), 104-109.
Cox, R. H., Martens, M. P. \& Russell, W. D. (2003). Measuring anxiety in athletics: The Revised Competitive State Anxiety Inventory-2. Journal of Sport Eु Exercise Psychology, 25(4), 519-533.

Cox, A. \& Williams, L. (2008). The roles of perceives teacher support, motivational climate and psychological need satisfaction in students` physical education motivation. Journal of Sport $\mathcal{E}$ Exercise Psychology, 30(2), 222-239.

Csikszentmihalyi, M. (1990). Flow: The psychology of optimal experience. New York: Harper \& Row.

Deci, E. L. \& Ryan, R. M. (2000). The "what" and the "why" of goal pursuits: Human needs and the selfdetermination of behaviour. Psychological Inquiry, 11(4), 227-268.

Fructuoso, C. \& Gómez, C. (2001). La danza como elemento educativo en el adolescente. Educación Física y Deportes, 66, 31-37.

García-Calvo, T., Cervelló, E., Jiménez, R., Iglesias, D. \& Moreno, J. A. (2010). Using self-determination theory to explain sport persistence and dropout in adolescent athletes. Spanish Journal of Psychology, 13(2), 677-684.

García-Calvo, T., Jiménez, R., Santos-Rosa, F., Reina, R. \& Cervelló, E. (2008). Psychometric properties of Spanish version of the Flow State Scale. Spanish Journal of Psychology, 11(2), 660-669.

García-Calvo, T., Sánchez-Oliva, D., Sánchez-Miguel, P. A., Leo, F. M. \& Amado, D. (2012). Análisis del grado de diversión e intención de persistencia en jóvenes deportistas desde la perspectiva de la teoría de la autodeterminación. Revista de Psicología del Deporte, 21(1), 7-13.

González-Cutre, D., Martínez Galindo, C., Alonso, N., Cervelló, E., Conte, L. \& Moreno, J. A. (2007). Las creencias implícitas de habilidad y los mediadores psicológicos como variables predictoras de la motivación autodeterminada en deportistas adolescentes. En J. Castellano \& O. Usabiaga (Eds.), Investigación en la actividad física y el deporte (Vol. 2, pp. 407-417). Vitoria: Universidad del País Vasco. González-Cutre, D., Sicilia, A. \& Moreno, J. A. (2008). Modelo cognitivo-social de la motivación de logro en educación física. Psicothema, 20(4), 642-651.

González-Cutre, D., Sicilia, A. \& Moreno, J. A. (2009). Dispositional flow in physical education: Rela- 
tionships with motivational climate, social goals, and perceived competence. Journal of Teaching in Physical Education, 28(4), 422-440.

Hair, J. F., Anderson, R. E., Tatham, R. L. \& Black, W. C. (1998). Multivariate data analysis. New Jersey: Prentice-Hall.

Hefferon, K. M. \& Ollis, S. (2006). 'Just clicks': An interpretative phenomenological analysis of professional dancers' experience of flow. Research in Dance Education, 7(2), 141-159.

Hu, L. \& Bentler, P. M. (1995). Evaluating model fit. En R. H. Hoyle (Ed.), Structural equation modeling: Concepts, issues, and applications (pp. 76-99). Thousand Oaks, CA: Sage.

Hu, L. \& Bentler, P. M. (1999). Cutoff criteria for fit indexes in covariance structure analysis: Conventional criteria versus new alternatives. Structural Equation Modeling, 6(1), 1-55.

Jackson, S. A. \& Csikszentmihalyi, M. (1999). Flow in sports: The keys to optimal experiences and performances. Champaign, IL: Human Kinetics.

Jackson, S. A., Kimiecik, J. C., Ford, S. \& Marsh, H. W. (1998). Psychological correlates of flow in sport. Journal of Sport and Exercise Psychology, 20(4), 358-378.

Jackson, S. A., Thomas, P. R., Marsh, H. W. \& Smethurst, C. J. (2001). Relationships between flow, self-concept, psychological skills, and performance. Journal of Applied Sport Psychology, 13(2), 129-153.

Kirchner, J. M., Bloom, A. J. \& Skutnick-Henley, P. (2008). The relationship between performance anxiety and flow. Medical Problems of Performing Artists, 23(2), 59-65.

Krane, V., Joyce, D. \& Rafeld, J. (1994). Competitive anxiety, situation criticality and softball performance. The Sport Psychologist, 8(1), 58-72.

Lim, B. S. C. \& Wang, C. K. (2009). Perceived autonomy support, behavioural regulations in physical education and physical activity intention. Psychology for Sport and Exercise, 10(1), 52-60.

López-Torres, M. R., Torregrosa, M. \& Roca, J. (2007). Características del flow, ansiedad y estado emocional, en relación con el rendimiento de deportistas de elite. Cuadernos de Psicología del Deporte, 7(1), 25-44.
Lowenthal, K. M. (2001). An introduction to psychological test and scales (2a. ed.). London: UCL Press.

Maehr, M. L. (1984). Culture and achievement motivation. American Psychologist, 29(12), 887-896.

Markland, D. \& Tobin, V. (2004). A modification to the Behavioral Regulation in Exercise Questionnaire to include an assessment of amotivation. Journal of Sport $\mathcal{E}$ Exercise Psychology, 26(2), 191-196.

Martens, R., Burton, D., Vealey, R. S., Bump, L. A. \& Smith, D. E. (1990). Development and validation of the Competitive State Anxiety Inventory-2 (CSAI-2). En R. Martens, R. S. Vealey \& D. Burton (Eds.), Competitive anxiety in sport (pp.117-190). Champaign, IL: Human Kinetics.

Martin, J. \& Cutler, K. (2002). An exploratory study of flow and motivation in theater actors. Journal of Applied Sport Psychology, 14(4), 344-352.

Maccallum, R. C. \& Austin, J. T. (2000). Applications of structural equation modeling in psychological research. Annual Review of Psychology, 51(1), 201-226.

Montero, C. (2010). Un análisis de la motivación en judo desde la Teoría de Autodeterminación. Tesis doctoral, Universidad Miguel Hernández de Elche, Comunidad Valenciana, España.

Moreno, J. A., Cano, F., González-Cutre, D., Cervelló, E. $\&$ Ruiz, L. M. (2009). Flow disposicional en salvamento deportivo: una aproximación desde la teoría de la autodeterminación. Revista de Psicología del Deporte, 18(1), 23-35.

Moreno, J. A., Cervelló, E. \& González-Cutre, D. (2006). Motivación autodeterminada y flujo disposicional en el deporte. Anales de Psicología, 22(2), 310-317.

Moreno, J. A., Cervelló, E. \& González-Cutre, D. (2010). The achievement goal and self-determination theories as predictors of dispositional flow in young athletes. Anales de Psicología, 26(2), 390-399.

Moreno, J. A., Cervelló, E. M. \& Martínez, A. (2007). Measuring self-determination motivation in a physical fitness setting: validation of the Behavioral Regulation in Exercise Questionnaire-2 (BREQ-2) in a Spanish sample. The Journal of Sport Medicine and Physical Fitness, 47(3), 366-378.

Moreno, J. A., López, M., Martínez Galindo, C. M., Alonso, N. \& González-Cutre, D. (2007). Validación preliminar de la escala de percepción del Clima Motivacional de los Iguales (CMI) y la es- 
cala de las Orientaciones de Meta en el Ejercicio (GOES) con practicantes españoles de actividades físico-deportivas. Revista Iberoamericana de Psicología del Ejercicio y el Deporte, 1(2), 13-28.

Moreno, J. A., López de San Román, M., Martínez Galindo, C., Alonso, N. \& González-Cutre, D. (2008). Peers' influence on exercise enjoyment: A selfdetermination theory approach. Journal of Sports Science and Medicine, 7(1), 23-31.

Newton, M., Duda, J. \& Yin, Z. (2000). Examination of the psychometric properties of the Perceived Motivational Climate in Sport Questionnaire-2 in a sample of female athletes. Journal of Sport Sciences, 18(4), 275-290.

Nordin-Bates, S. M., Quested, E., Walker, I. J. \& Redding, E. (2012). Climate change in the dance studio: Findings from the UK Centres for Advanced Training. Sport, Exercise, and Performance Psychology, 1(1), 3-16.

Ntoumanis, N. (2005). A prospective study of participation in optional school physical education using a self-determination theory framework. Journal of Educational Psychology, 97(3), 444-453.

Ntoumanis, N. \& Vazou, S. (2005). Peer motivational climate in youth sport: Measurement development and validation. Journal of Sport $\mathcal{E}$ Exercise Psychology, 27(4), 432-455.

Nunnally, J. C. \& Bernstein, I. H. (1994). Psychometric theory (3a. ed.). New York: McGraw-Hill.

Ommundsen, Y. \& Kval $\varnothing$, S. E. (2007). Autonomy-mastery, supportive or performance focused? Different teacher behaviours and pupils' outcomes in physical education. Scandinavian Journal of Educational Research, 51(4), 385-413.

Quested, L. \& Duda, J. L. (2009). Perceptions of the motivational climate, need satisfaction, and indices of well- and ill-being among hip hop dancers. Journal of Dance Medicine and Science, 13(1), 10-19.

Quested, L. \& Duda, J. L. (2010). Exploring the socialenvironmental determinants of well- and ill-being in dancers: A test of basic needs theory. Journal of Sport $\mathcal{E}$ Exercise Psychology, 32(1), 39-60.

Reinboth, M. \& Duda, J. L. (2006). Perceived motivational climate, need satisfaction, and indices of well-being in team sports: A longitudinal perspective. Psychology of Sport Eु Exercise, 7(3), 269-286.
Russell, W. D. (2001). An examination of flow state occurrence in college athletes. Journal of Sport Behavior, 24(1), 83-107.

Ryan, R. M. \& Deci, E. L. (2000). Self-determination theory and the facilitation of intrinsic motivation, social development and wellbeing. American Psychologist, 55(1), 68-78.

Sicilia, A., Águila, C., González-Cutre, D. \& MorenoMurcia, J. A. (2011). Factores motivacionales y experiencia autotélica en el ejercicio físico: propuesta de un modelo explicativo. Universitas Psychologica, 10(1), 125-135.

Sproule, J., Wang, C. K. J., Morgan, K., McNeills, M. \& McMorris, T. (2007). Effects of motivational climate in Singaporean physical education lessons on intrinsic motivation and physical activity intention. Personality and Individual Differences, 43(5), 1037-1049.

Standage, M., Duda, J. L. \& Ntoumanis, N. (2003). A model of contextual motivation in physical education: Using constructs from self-determination and achievement goal theories to predict physical activity intentions. Journal of Educational Psychology, 95(1), 97-110.

Standage, M., Duda, J. L. \& Pensgaard, A. M. (2005). The effect of competitive outcome and task-involving, ego-involving, and cooperative structures on the psychological well-being of individuals engaged in a co-ordination task: A self- determination approach. Motivation and Emotion, 29(1), 41-68.

Taylor, J. \& Taylor, C. (1995). Psychology of dance. Champaign, IL: Human Kinetics.

Tessier, D., Sarrazin, P. \& Ntoumanis, N. (2010). The effect of an intervention to improve newly qualified teachers` interpersonal style, students motivation and psychological need satisfaction in sport-based physical education. Contemporary Educational Psychology, 35(4), 242-253.

Thogersen-Ntoumani, C. \& Ntoumanis, N. (2006). The role of self-determined motivation in the understanding of exercise-related behaviours, cognitions and physical self-evaluations. Journal of Sports Sciences, 24(4), 393-404.

Ulrich-French, S. \& Smith, A. L. (2009). Social and motivational predictors of continued youth sport 
participation. Psychology of Sport and Exercise, 10(1), 87-95.

Valle, A., Rodríguez, S., Cabanach, R. G., Núñez Pérez, J. C., González-Pienda, J. A. \& Rosario, P. (2009). Perfiles motivacionales y diferencias en variables afectivas, motivacionales y de logro. Universitas Psychologica, 9(1), 109-121.

Vallerand, R. J. (2001). A hierarchical model of intrinsic and extrinsic motivation in sport and exercise. En G. C. Roberts (Ed.), Advances in motivation in sport and exercise (pp. 263-319). Champaign, IL: Human Kinetics.

Vallerand, R. J. (2007). Intrinsic and extrinsic motivation in sport and physical activity. En G. Tenenbaum \& R. C. Eklund (Eds.), Handbook of sport psychology (3a. ed., pp. 59-83). New York: Wiley.
Vergara, G. (2007). La experiencia estética en el pensamiento de Roman Ingarden. Cultura. Internacional Journal of Philosophy of Culture and Axiology, 8(7), 117-136.

Weiss, M. R., Amorose, A. J. \& Wilko, A. M. (2009). Coaching behaviors, motivational climate, and psychosocial outcomes among female adolescent athletes. Pediatric Exercise Science, 21(4), 475-492.

Yli-Piipari, S., Watt, A., Jaakkola, T., Liukkonen, J. \& Nurmi, J. E. (2009). Relationships between physical education students' motivational profiles, enjoyment, state anxiety, and self-reported physical activity. Journal of Sports Science and Medicine, 8(3), 327-336. 